\title{
REDES MODULARES DE INOVAÇÃO EM INDÚSTRIAS DE ALTA TECNOLOGIA
}

\section{MODULAR INNOVATION NETWORKS IN HIGH-TECHNOLOGY INDUSTRIES}

\section{REDES MODULARES DE INNOVACIÓN EN INDUSTRIAS DE ALTA TECNOLOGÍA}

\author{
Milton Freitas Chagas Junior \\ Doutor em Engenharia Aeronáutica e Mecânica pelo Instituto Tecnológico de Aeronáutica - ITA \\ Professor e Pesquisador da Universidade Nove de Julho - UNINOVE \\ E-mail: miltonfc@uninove.br (Brasil)
}

\section{Roque Rabechini Jr}

Doutor em Engenharia de Produção pela Escola Politécnica da Universidade de São Paulo POLI/USP

Professor do Programa de Mestrado Profissional em Administração - Gestão de Projetos da Universidade Nove de Julho - MPA-GP/UNINOVE

E-mail: roquejr@uninove.br (Brasil)

\section{Arnoldo Souza Cabral \\ Doutor em Ciências pelo Instituto Tecnológico de Aeronáutica - ITA \\ Professor do Instituto Tecnológico de Aeronáutica - ITA \\ E-mail: cabral@ita.br (Brasil)}

\section{Milton de Abreu Campanario}

Doutor em Filosofia pela Cornell University, EUA

Coordenador do Programa de Mestrado e Doutorado em Administração da Universidade Nove de Julho - PMDA/UNINOVE

E-mail: miltonac@uninove.br (Brasil) 
Redes Modulares de Inovação em Indústrias de Alta Tecnologia

\title{
REDES MODULARES DE INOVAÇÃO EM INDÚSTRIAS DE ALTA TECNOLOGIA
}

\section{RESUMO}

Esse trabalho mostra como estão estruturadas as redes interorganizacionais envolvendo duas organizações representantes de indústrias de alta tecnologia: a Embraer e a Siemens. Foram estudados três casos: o projeto dos jatos ERJ 170 / 190, da Embraer, e os equipamentos de raio X e da plataforma SX, da Siemens. Seu objetivo foi expandir a compreensão sobre o conceito de integração de sistemas no que se refere às atividades de projetos. Adotou-se a metodologia de estudo de caso. Os dados foram levantados por meio de entrevistas e análise documental nas duas empresas. Foi possível constatar como estão estruturadas as redes interorganizacionais das empresas estudadas, analisados sob o prisma de tipos de acoplamentos em projetos propostos pela teoria e verificados por esse estudo. Dessa forma, descobriu-se o posicionamento dos casos estudados, como sendo, dois acoplamentos frouxos e um forte. Além disso, verificou-se também que as organizações que lideram as redes modulares de inovação são, em última instância, integradoras de conhecimento.

Palavras-chave: Gerenciamento de Projetos; Inovação; Integração de Sistemas; Redes Modulares.

\section{MODULAR INNOVATION NETWORKS IN HIGH-TECHNOLOGY INDUSTRIES}

\begin{abstract}
The article shows how interorganizational modular networks are structured in two high-tecnology industries: Embraer and Siemens. Three product platforms were used in the research: the Embraer 170/190 program from Embraer and the SX platform and X-ray equipment from Siemens. The objective of this article is to expand our comprehension of systems integration as a coordination mechanism in projects. The method adopted in the research is a case study. Data were collected by interviews and by analysis of documents from the two organizations. It was possible to identify and analyze the influence of the level of coupling and how the networks are structured. The research shows two levels of coupling in the modular innovation networks studied: two loosely coupled networks and one tightly coupled network. In addition, it was verified that the system integrators, the organizations that lead the modular networks, act as a matter of facts as knowledge integrators.
\end{abstract}

Keywords: Innovation; Modular Networks; Project Management; Systems Integration.

Revista Ibero-Americana de Estratégia - RIAE, São Paulo, v. 10, n. 3, p. 173-198, set./dez. 2011. 
REDES MODULARES DE INNOVACIÓN EN INDUSTRIAS DE ALTA TECNOLOGÍA

\section{RESUMEN}

Este trabajo muestra cómo están estructuradas las redes interorganizacionales involucrando dos organizaciones de industrias de alta tecnología: la Embraer y Siemens. Fueron estudiados tres casos: el proyecto de los aviones de pequeño porte ERJ 170 / 190, de la Embraer, y los equipamientos de rayos X y de la plataforma SX, de Siemens. El objetivo fue ampliar la comprensión sobre el concepto de integración de sistemas en lo que se refiere a las actividades de proyectos. Se adoptó la metodología del estudio de caso. Los datos fueron levantados por medio de las entrevistas y el análisis documental en las dos empresas. Fue posible constatar cómo están estructuradas las redes interorganizacionales de las empresas estudiadas teniendo como base los tipos de acoplamientos propuestos por la teoría y verificados por este estudio. De esta forma, se descubrió el posicionamiento de los casos estudiados, en los cuales, dos acoplamientos fueron débiles y uno fuerte. Además, también se verificó que las organizaciones que lideran las redes modulares de innovación son, en última instancia, integradoras de conocimiento.

Palabras-clave: Administración de Proyectos; Innovación; Integración de Sistemas; Redes Modulares. 


\section{INTRODUÇÃO}

O campo de estudo das organizações encontra duas correntes do pensamento teórico/conceitual que têm contribuído para a formação de modelos de estrutura organizacional que auxiliam a entender a realidade das empresas e como elas influenciam suas ações. A primeira corrente relaciona-se à organização industrial, tendo suas bases sido lançadas por Coase por meio de seu artigo seminal: "A natureza da firma", que estudou as organizações do ponto de vista da economia dos custos de transação. Essa abordagem considera a existência de outros custos, além dos tradicionais custos de produção, da tradição neoclássica, e considera as organizações, não como uma função de produção, mas como um conjunto de contratos coordenados, que levam à execução da função produtiva. Seguindo a tradição de Coase, Williamson (1996) expandiu os entendimentos sobre a definição dos limites - ou fronteiras - da firma por meio da coordenação vertical da produção.

A segunda corrente, proposta por Lawrence e Lorsch (1967), apresentou os conceitos de estruturas a partir das variáveis diversificação e integração, como elementos de análise das atividades da firma para atender às necessidades organizacionais de seus mercados/clientes.

Para Lawrence e Lorsch (1967), as formas estruturais das organizações são influenciadas pelas mudanças impostas por diferentes fatores - tecnologia, ciência e mercado. Num ambiente instável, de elevada incerteza tecnológica, de alta diferenciação, a organização deve ser preparada para o desenvolvimento de projetos em consonância com suas atividades rotineiras. Para Mintzberg et al. (2000), no entanto, as organizações devem reagir às formas estruturais convencionais e adotar estruturas em redes, fazendo com que a organização se interconecte com outras organizações, tais como universidades, outras empresas, instituto de pesquisas, entre outras. O fato é que a partir dos estudos de Mintzberg et al. (2000) novas visões sobre as formas organizacionais surgiram.

Tradicionalmente, a pesquisa sobre a economia dos custos de transação considera que a coordenação das atividades econômicas é atingida por meio do sistema de preços - trocas de mercado - ou pelo exercício da autoridade, através de firmas integradas verticalmente. Coase argumenta que o fator crítico para a seleção entre os dois mecanismos de coordenação é baseado, fundamentalmente, nos custos de transação. Esses dois casos - sistemas de preços e integração vertical - são casos extremos de um espectro amplo de variação, que são considerados híbridos, pois mesclam características desses casos externos. Williamson (1996) propôs um conjunto de atributos que influenciavam os custos de transação, considerados decisivos para definir o modo apropriado de governança. Esses atributos da transação são: especificidade dos ativos, incerteza,

Revista Ibero-Americana de Estratégia - RIAE, São Paulo, v. 10, n. 3, p. 173-198, set./dez. 2011. 
frequência com que essa transação ocorre em determinado período de tempo. Esses atributos são considerados, na definição do modelo, por hipóteses comportamentais e cognitivas: o oportunismo e a racionalidade limitada, respectivamente. Para a definição das fronteiras da firma, o atributo principal, para Williamson, é a especificidade de ativos.

Esses dois casos extremos de mecanismos de coordenação, discutidos pela economia dos custos de transação, se tornaram o foco de pesquisas empíricas que estudavam os mecanismos de coordenação em ambientes caracterizados por elevadas taxas de mudança tecnológica. Muitos teóricos, tais como Afuah (2001) e Teece (1996), sustentam que, nesses ambientes, o mecanismo apropriado de coordenação é a firma integrada verticalmente, enquanto as trocas de mercado são mais apropriadas para ambientes estáveis. Outros pesquisadores sustentam posições diversas, salientando que a integração vertical não é um mecanismo apropriado de coordenação quando as mudanças tecnológicas tornam obsoletas as capacitações centrais das firmas. Podendo contar com a diversidade das trocas de mercado, as firmas podem explorar relacionamentos com diversos fornecedores em vez de se restringir apenas a um único relacionamento interno (Arora et al.,1998).

Com a difusão das tecnologias relacionadas com o atual paradigma técnico-econômico (Dosi,1988), as tecnologias de informação e telecomunicações (ICT), houve uma redefinição dos custos de transação e das formas de governança entre agentes econômicos, pois as redes interorganizacionais se tornaram a forma dominante da organização de diversas indústrias, principalmente aquelas marcadas pelo forte caráter inovador. Em consequência, muitas firmas que eram integradas verticalmente passaram a ser integradoras das atividades desenvolvidas por outras organizações. Para tentar entender essa tendência, muitas pesquisas vêm sendo desenvolvidas com a finalidade de compreender a natureza dos mecanismos de coordenação de relacionamentos em rede que mesclam atributos do sistema de preços, característico dos mercados, e da integração vertical, característica da autoridade exercida por meio de relacionamentos hierárquicos. Essa pesquisa é um esforço nessa direção.

Baseado em dois estudos de caso de firmas que estabelecem e lideram redes interorganizacionais de abrangência global - Embraer e Siemens Brasil - o objetivo desse trabalho foi expandir a compreensão sobre o conceito de integração de sistemas, entendido como um mecanismo de coordenação intermediário entre o sistema de preços e a integração vertical. Para atingir esse objetivo, uma questão de pesquisa norteadora foi estabelecida: quais as formas de coordenação de redes interorganizacionais tendo a teoria de tipos de acoplamento em projetos como referência?

Em particular, foram analisados os casos de indústrias multitecnológicas, onde se pôde notar

Revista Ibero-Americana de Estratégia - RIAE, São Paulo, v. 10, n. 3, p. 173-198, set./dez. 2011. 
a presença de firmas líderes, que são responsáveis pela coordenação e integração dos processos de inovação. Seguindo a definição proposta inicialmente por Rothwell (1992), essas firmas agem como integradoras de sistemas de suas indústrias, e nesse contexto, sistemas se referem tanto aos produtos finais quanto as organizações que participam dessas redes. Será utilizada a estrutura conceitual proposta por Brusoni, Prencipe and Pavitt (2001), que considera duas variáveis - previsibilidade do comportamento do produto depois da integração final e taxas de mudança técnica - como determinantes do grau de acoplamento entre agentes das redes, determinando três formas de relacionamentos interorganizacionais em redes: relacionamentos desacoplados, de acoplamento frouxo e firme. Essas formas de relacionamento entre agentes de rede são caracterizadas por mecanismos de coordenação interorganizacionais distintos - sistemas de preços, integração de sistemas e integração vertical, respectivamente.

Esse trabalho está organizado da forma seguinte: a seção 2, descreve a estrutura conceitual de análise, sob a ótica da organização das atividades de projetos na formação de redes interorganizacionais. A seção 3, apresenta o método da pesquisa adotado nesse trabalho: o estudo de caso. A seção 4, faz a caracterização das organizações objeto dessa pesquisa. A seção 5, aprofunda o conceito de integração de sistemas como um mecanismo intermediário entre sistema de preços e a integração vertical, e analisa os desdobramentos da estrutura conceitual para os estudos de caso. A seção 6, apresenta as conclusões do trabalho.

\section{A ESTRUTURAÇÃO DAS ATIVIDADES DE PROJETOS POR REDES INTERORGNIZACIONAIS: A BASE TEÓRICA REVISTA}

A globalização tem imposto desafios importantes para as organizações, no que se refere à sustentabilidade competitiva. Nesse contexto, a amplitude de alternativas é significativa e vai desde o estabelecimento do sistema de preços adotado em seus produtos até a forma organizacional adequada para se apresentar ao mercado com eficácia.

A segmentação e a integração das atividades de uma organização são determinantes para a viabilização de implementação de seus objetivos estratégicos (Deresky, 2004 e Vasconcellos e Hemsley, 2002). O quadro de alternativas estruturais que se apresenta, decorrente dessas duas variáveis, mescla as formas tradicionais de organização: as hierárquicas e os mercados . No entanto, com a intensificação das atividades de projetos nas empresas, novas preocupações se apresentam na análise das formas organizacionais. A busca por estruturas organizacionais

Revista Ibero-Americana de Estratégia - RIAE, São Paulo, v. 10, n. 3, p. 173-198, set./dez. 2011. 
inovadoras que apresentem baixo nível de formalização, que possibilitem multiplicidade de comando, a diversificação elevada e comunicação horizontal e diagonal, têm sido frequente entre as empresas que precisam conjugar atividades rotineiras e não-rotineiras, visando a criação e a manutenção de vantagens competitivas. Num ambiente inovador, onde as empresas necessitam realizar projetos que envolvam várias áreas funcionais, o uso da estrutura funcional é limitado. No passado, a estrutura funcional poderia satisfazer as necessidades das empresas, pois elas lidavam com um grau pequeno de diversificação de produtos. Com a necessidade de diversificar e integrar tecnologias no desenvolvimento de novos produtos e processos, as empresas passaram a buscar novas formas organizacionais que pudessem responder a esses desafios, com o intuito de buscar uma maior integração e resolver os problemas existentes (Patah e Carvalho, 2002).

No entanto, a organização projetizada em si,está restrita à organização. A literatura dedicada ao campo de estudos de gerenciamento de projetos não tem proposto novas alternativas estruturais, apenas constatam as formas já existentes (Kerzner, 2001; Cleland, 2007; Gray e Larson, 2009; Carvalho e Rabechini JR., 2007).

Para Shenhar (2001), as pesquisas que mais influenciaram a teoria da contingência foram a de Lawrence e Lorsch (1967), Perrow (1967) e Thompson (1967). Enquanto Lawrence e Lorsch (1967) analisavam como diferentes taxas de alteração em tecnologias, ciências e mercado impactavam a habilidade das organizações em lidar com essas mudanças, Thompson (1967) alertava para o fato de que a incerteza constituía o principal desafio das organizações complexas, cujas principais fontes de incerteza são a tecnologia e o meio ambiente. Perrow (1967), por sua vez, conclui que as dimensões incerteza e complexidade são as mais citadas na literatura, com predominância da incerteza tecnológica, a partir de um ponto de vista integrado em tecnologia e organizações complexas.

A organização por projeto, no limite, pode ser um caminho para uma nova possibilidade de estruturação, denominada redes intereorganizacionais (Hobday, 1998). A estrutura de uma rede interorganizacional pode guiar a empresa a um mercado competitivo específico (Kogut, 2000), bem como melhorar o desempenho gerencial quando se trata de empresas com conhecimentos heterogêneos (Rodan e Galunic, 2004), resultantes de diferentes especializações.

A coordenação e integração das atividades executadas por agentes econômicos articulados em redes é uma parte essencial do processo de inovação. No caso específico das indústrias multitecnológicas, as firmas que lideram esses processos devem ter um estoque de conhecimento interno maior do que o necessário para executar as atividades produtivas que desenvolvem. As razões que explicam a necessidade de manutenção desse estoque de conhecimento em excesso são

Revista Ibero-Americana de Estratégia - RIAE, São Paulo, v. 10, n. 3, p. 173-198, set./dez. 2011. 
duas: lidar com o desequilíbrio técnico e com as interdependências sistêmicas no nível do produto.

As diferenças entre as taxas de mudança técnica das disciplinas que fundamentam um produto podem causar o que Rosenberg (1976) chamou de technical imbalance, ou desequilíbrio técnico. Isso acontece quando a taxa de mudança técnica de uma parte do sistema é muito maior que a do sistema como um todo, exigindo assim mais atenção aos melhoramentos complementares do restante do sistema. A partir daí, surgiu o conceito de saliência. Fazendo uma metáfora com ações militares, uma divisão que avança mais rápido que as demais cria uma saliência. É aí que a integração deve atuar. Deve-se descobrir se a melhor estratégia é acelerar todas as divisões ou desacelerar a divisão que avança mais rápido, para evitar a fragilidade do conjunto. Dito de outra forma, a saliência cria um problema de desempenho diferenciado que pode se repercutir em todo o sistema e, portanto, deve-se buscar uma solução que traga o sistema para uma situação de equilíbrio. Isso só pode ser feito pela coordenação consciente de quem possui domínio geral do sistema.

A interdependência sistêmica dos produtos multitecnológicos exige que a otimização do sistema seja feita considerando-se todos os componentes e subsistemas, integrados em determinada configuração. A otimização isolada de componentes e subsistemas, não faz sentido. Além do mais, existem as interdependências imprevisíveis que geram sérios problemas aos produtos multitecnológicos, como por exemplo, as mecânicas: vibração e ressonância, que só podem ser percebidas por meio da prototipação, ou mesmo numa fase mais avançada do ciclo de vida do produto, como o uso do produto em seu ambiente operacional.

Os determinantes da forma de acoplamento entre a organização líder e outros agentes da rede são: interdependências do produto - previsível ou imprevisível - e da taxa de mudança de componentes tecnológicos - constante e inconstante.Estes determinantes têm implicações em termos da contratação de atividades de $\mathrm{P} \& \mathrm{D}$, projeto detalhado e produção, e o principal mecanismo de coordenação das atividades entre agentes da rede.

No caso de produtos baseados em estratégias modulares, a coordenação das atividades se refere aos agentes das redes contratados para execução do projeto detalhado e a produção dos módulos, ou fornecedores especializados de primeiro nível. , As firmas líderes estabelecem redes em torno de produtos de arquitetura modular e se beneficiam das vantagens tanto da integração quanto da diversificação, resultante dos diferentes fornecedores especializados.

As firmas líderes traduzem as necessidades e expectativas de potênciais clientes em requisitos que são utilizados na definição de uma arquitetura do produto que melhor atenda a essas necessidades e expectativas. Decompõe essa arquitetura em módulos e os contrata de fornecedores

Revista Ibero-Americana de Estratégia - RIAE, São Paulo, v. 10, n. 3, p. 173-198, set./dez. 2011. 
especializados de primeiro nível. Os integradores de sistemas fazem uso intensivo da contratação de atividades de $\mathrm{P} \& \mathrm{D}$, projeto detalhado e produção dos módulos para minimizar os riscos, custos e tempos de desenvolvimento de seus produtos, além de estabelecer relacionamentos de longo prazo que permitem a redução dos custos de transação, tornando o produto mais competitivo.

Depois de decompor o produto em módulos, os integradores de sistemas sincronizam seu trabalho com o dos fornecedores, para recompor o produto, assegurando sua consistência e desempenho, para atender aos requisitos dos clientes e de órgãos de regulação.

\section{MÉTODO DA PESQUISA}

Esse trabalho apresenta as características de um estudo exploratório, na medida em que busca a expansão da compreensão de um problema geral. Para abordá-lo, decidiu-se pelo método do estudo de caso. Esse método é indicado para análise de casos específicos, considerados representativos de determinada situação, e válido quando se está buscando ampliar o entendimento sobre determinado tema, e apresenta como alternativa adequada para organizar as informações e tratá-las de forma sistemática. Com essa estratégia de pesquisa, esperou-se ter aprofundado a análise sobre a disciplina estudada e explorada nesse trabalho, bem como propiciar para estudantes e praticantes interessados no assunto, a utilizarem seu conteúdo e suas conclusões (Yin, 2001).

A seleção da unidade de análise foi intencional, pois, tratava-se de duas organizações que, supostamente, apresentavam as características de utilização de multitecnologias na constituição de seus produtos e de formação de redes interorganizacionais para o desenvolvimento das atividades de projetos. As empresas pesquisadas foram a Embraer, líder do mercado comercial da aviação regional a jato, e a Siemens, uma das empresas líderes do mercado de eletroeletrônico global. Uma breve apresentação dessas empresas será feita ao longo da exposição desse trabalho. A unidade de análise da Embraer foi o programa de desenvolvimento da família 170/190, composto pelo projeto de 4 aeronaves, dos quais um projeto será estudado com mais detalhes: o Embraer 170. Da Siemens, foram analisadas duas unidades: 1) eletromedicina, por meio do produto equipamentos de raio $\mathrm{X}$ e, 2) comunicação móvel, através da plataforma de redes de dados móveis SX.

O levantamento de informações qualitativas, a partir de fontes primárias, foi feito por meio de entrevistas com gestores das áreas de tecnologia corporativa das duas empresas analisadas nesse estudo, além de gestores das áreas de desenvolvimento de produtos. Os entrevistados foram questionados sobre a forma de contratação externa das atividades de $\mathrm{P} \& \mathrm{D}$, projeto detalhado e

Revista Ibero-Americana de Estratégia - RIAE, São Paulo, v. 10, n. 3, p. 173-198, set./dez. 2011. 
produção e também sobre fatores que influenciam a decisão entre fazer internamente ou comprar, competências-chave necessárias para a coordenação e integração de sistemas e as estratégias utilizadas para aumentar a competitividade de produtos baseados em arquitetura modular. Foram também analisados relatórios sobre as áreas tecnológicas e de desenvolvimento de produtos, de interesse dessa pesquisa, com a finalidade de obter informações complementares. $\mathrm{O}$ foco em tais informações qualitativas restringiu o campo de observação, fortificando a ideia de estudo de caso que, para Eisenhardt (1989), esse tipo de estudo centraliza sua atenção no entendimento de dinâmicas presentes em realidades particulares.

Em ambos os casos, os dados qualitativos foram analisados seguindo a lógica indutiva, ou seja, partiu-se de um raciocínio que considerou a análise de eventos particulares até se chegar a fatos gerais.

Para esclarecer melhor os procedimentos metodológicos adotados nesse caso, foi desenvolvido um processo de pesquisa (Figura 1).

Figura 1- Diagrama representativo da metodologia de pesquisa do estudo de caso.

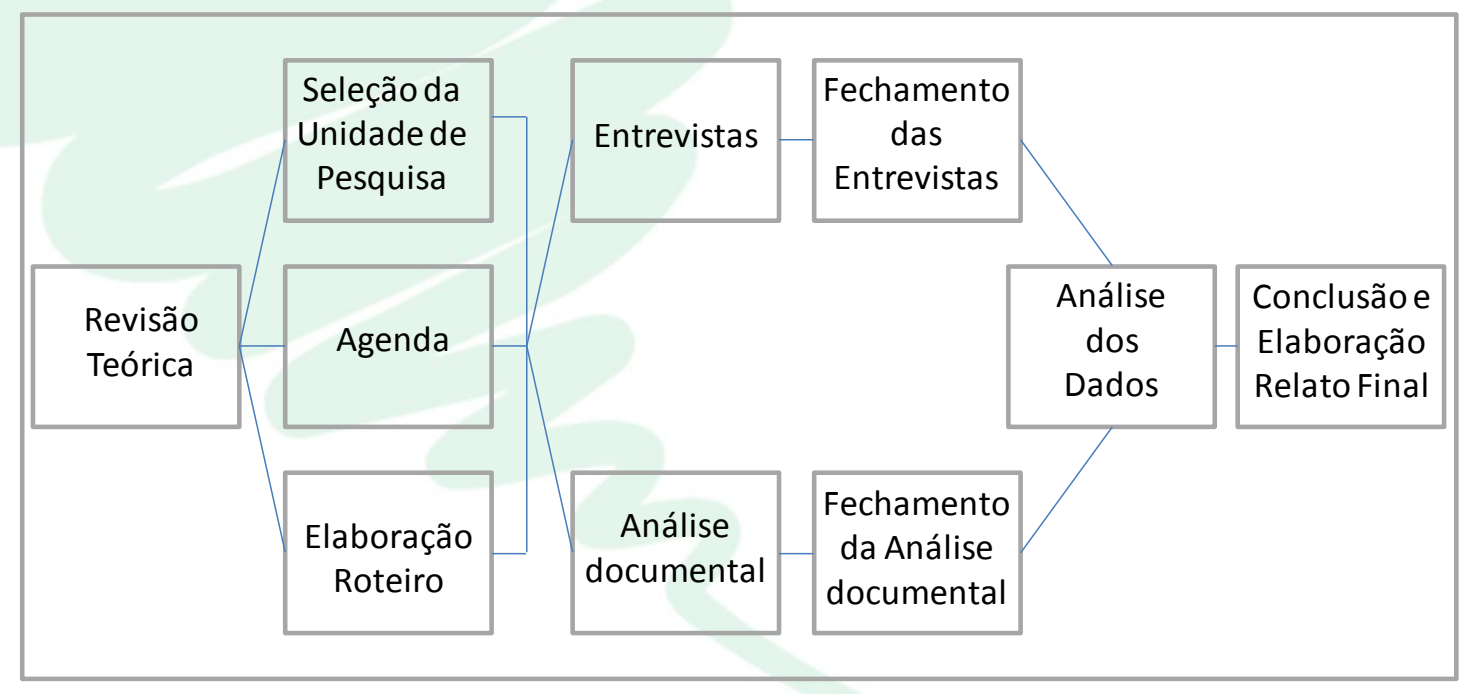

Fonte: Elaborado pelos autores.

É possível observar que a pesquisa foi desenvolvida, considerando-se inicialmente, a revisão teórica sobre o assunto, envolvendo a literatura sobre economia das organizações e formas organizacionais das atividades de projetos. Uma agenda do estudo de caso, então, foi construída visando a selecionar os entrevistados e orientar os pesquisadores na condução das entrevistas. Com isso, um roteiro de entrevista foi confeccionado e aplicado nas entrevistas. Em linhas gerais, esse roteiro teve como informações: identidade dos entrevistados, identificação do projeto e/ou produto

Revista Ibero-Americana de Estratégia - RIAE, São Paulo, v. 10, n. 3, p. 173-198, set./dez. 2011. 
respectivo, levantamento de dados sobre a coordenação das atividades do integrador da rede, dados sobre a relação com os fornecedores, tipo de segmentação e níveis de integração, entre outras.

Em paralelo foi possível acessar documentos não-confidenciais das empresas para obtenção de mais dados, bem como para comprovação das informações declaradas pelos entrevistados.

Uma vez de posse das informações, os pesquisadores procederam a análise dos dados e, por fim, desenharam o caso. Vale a pena ressaltar que a partir do entendimento do caso foi possível posicionar os casos estudados na matriz que determina a forma de acoplamento e a estrutura de rede interorganizaional na qual os projetos foram gerenciados. Com as informações provenientes dessas pesquisas, foi possível entender o problema, bem como delimitar a abrangência do estudo. $\mathrm{O}$ caso será apresentado em seguida.

\section{CARACTERIZAÇÃO DAS ORGANIZAÇÕES: EMBRAER E SIEMENS BRASIL}

As unidades de pesquisa selecionadas nesse trabalho foram escolhidas a partir de duas empresas que se caracterizam por serem líderes de redes interorganizacionais no desenvolvimento de projetos e produtos. A apresentação dessas empresas, bem como as respectivas unidades de análise de pesquisa, será feita a seguir.

\section{a. Embraer}

A partir da implantação bem-sucedida de um plano estratégico de longo prazo, que permitiu a criação e desenvolvimento uma sólida base científica e educacional, a indústria aeronáutica brasileira vem acumulando capacitações tecnológico-industriais, geradas endogenamente e obtidas e incorporadas por meio de fontes externas (Chagas Junior e Cabral 2010).

O apoio estatal foi fundamental para a formação de equipes de engenharia com competências-chave, para conceber, desenvolver, certificar e produzir aeronaves. O governo brasileiro, considerando que indústria aeronáutica tinha importância estratégica para o país, contratou, em 1945, por meio do ministério da aeronáutica, o professor Richard H. Smith, do Massachusetts Institute of Technology (MIT), para o desenvolvimento do plano geral de criação do Centro Técnico Aeroespacial (CTA). Em 1946, foi instalada a comissão organizadora para a criação do CTA, dirigida pelo Coronel-Aviador, Casimiro Montenegro Filho.

Em 1950, o CTA iniciou suas atividades com o Instituto Tecnológico de Aeronáutica (ITA) que formou grande parte da massa crítica transferida à Embraer. A grande preocupação com 
pesquisa básica, pesquisa aplicada e desenvolvimento experimental permitiu a criação de sólidas capacitações científicas e tecnológicas, que aliadas a uma política industrial vertical, incluindo importantes compras governamentais, possibilitaram o desenvolvimento de uma estratégia tecnológica muito bem-sucedida, e sem precedentes na história do país. Essa estratégia possibilitou a acumulação de capacitações tecnológicas, derivadas do contínuo esforço de P\&D. Com forte apoio do Estado, foi possível desenvolver a capacidade de inovar e absorver conhecimentos tecnológicos de diferentes procedências.

Aproximadamente 20 anos depois da criação do CTA, a Embraer foi criada. Ao longo dos anos 1970, utilizou, de forma intensa, fontes externas de tecnologia, para auxiliar e complementar suas capacitações científicas e tecnológicas, de produção e comerciais. A Embraer se desenvolveu numa época em que outras construtoras aeronáuticas se mostravam dispostas a licenciar tecnologias para uma pequena indústria de um país subdesenvolvido, com o objetivo claro de penetrar nos mercado brasileiro e sul-americano.

Hoje a Embraer lidera o mercado comercial de aviação regional a jato e é uma das principais exportadoras brasileiras. Com mais de 14 mil funcionários, entregou 148 aeronaves em 2004, o que é considerável para uma indústria caracterizada pela baixa escala de produção. Teve um faturamento da ordem de R $\$ 9$ bilhões, dos quais $80 \%$ são do segmento aviação regional.

Construtoras aeronáuticas de tradição na aviação comercial, como Bae Systems, Saab, Fairchild e Fokker foram forçadas a sair desse mercado em razão da intensa competição, entre a Embraer e a Bombardier.

A Embraer foi privatizada em 1994, depois de um longo período de crise financeira que teve início no final da década de 1980. ÁA partir de então, começou a utilizar as parceiras de risco em seus programas de desenvolvimento. No segmento da aviação regional, o custo de desenvolvimento de uma nova geração de aeronaves é da ordem de US\$1 bilhão. A utilização de mecanismos que permitam o compartilhamento dos riscos e custos de desenvolvimento - como as parcerias de risco - com fornecedores vem crescendo de maneira significativa na indústria.

Na Embraer, a evolução da rede de fornecedores e parceiros de risco levou a uma acentuada redução do número de fornecedores e ao aumento progressivo dos parceiros de risco . Nota-se que com o desenvolvimento da família ERJ-145, nos anos 1990, iniciou-se a utilização de desenvolvimento de produtos baseados em arquiteturas modulares com o progressivo aumento das atividades, de projeto detalhado e produção dos módulos, atribuídas aos parceiros de risco. Os relacionamentos entre parceiros não é baseado exclusivamente em critérios de preços, eles são de longo prazo e, por isso, exigem alinhamento de esforços de $\mathrm{P} \& \mathrm{D}$, além de investimento em ativos

Revista Ibero-Americana de Estratégia - RIAE, São Paulo, v. 10, n. 3, p. 173-198, set./dez. 2011. 
específicos para a produção dos módulos. Dessa forma, fornecedores especializados compartilham riscos e custos não recorrentes do desenvolvimento e produção de módulos. Na família Embraer 170/190, nota-se a consolidação da estratégia da modularidade com a atribuição das atividades de projeto detalhado e produção para parceiros de risco envolvendo todo o produto. A Embraer coordena o desenvolvimento dos trabalhos dos parceiros e fornecedores e se responsabiliza pela integração final das aeronaves. (Chagas Junior, 2005).

\section{b. Siemens Brasil}

A Siemens está fortemente representada no Brasil. As primeiras atividades da empresa no país datam de 1867, com a instalação da linha telegráfica pioneira entre o Rio de Janeiro e o Rio Grande do Sul. Em 1895, no Rio de Janeiro, foi aberto o primeiro escritório e, dez anos mais tarde, em 1905, ocorria a fundação da empresa no Brasil. A Siemens é uma das empresas líderes do mercado eletroeletrônico global, com atividades em segmentos de negócios que englobam telecomunicações, automação e controle, energia, transportes, medicina e iluminação.

As unidades de negócio, objeto dessa pesquisa, possuem como principais objetivos a busca constante pela inovação por meio do desenvolvimento de novos produtos, serviços e soluções, adequados às necessidades dos clientes, sejam indústrias de grande porte, hospitais, operadoras de telecomunicações, pequenas empresas ou, ainda, o usuário de aparelho telefônico de linha fixa e de telefone celular.

A área de comunicações da empresa detém hoje $56 \%$ do faturamento de $\mathrm{R} \$ 5,9$ bilhões e possui atualmente 2.118 colaboradores, distribuídos da seguinte forma: em quatro centros de pesquisa e desenvolvimento de hardware e software para produtos e soluções integradas de classe mundial, em duas unidades fabris que atendem a região Mercosul, em nove escritórios regionais e três escritórios de vendas localizados em todo o País.

Pelo alto dinamismo tecnológico dessa área, é nesse segmento de negócios que a Siemens Brasil concentra boa parte dos esforços em P\&D e inovação, tanto com equipes internas, quanto em parcerias com universidades e centros de pesquisa.

\section{i. Telecomunicações}

$\mathrm{Na}$ área de comunicações para operadoras, houve a consolidação de duas áreas de $\mathrm{P} \& \mathrm{D}$ muito atuantes no passado, quais sejam: comutação e transmissão, tendo realizado projetos de adaptação, nacionalização e substituição de importações. A partir de 1993, passou a ampliar suas equipes de desenvolvimento e consolidar sua capacidade, passando a conquistar centros de 
competência em desenvolvimento em: cartão de 16 canais de linha no mundo; centro de competência global para linecards; linecards específicos para os EUA e família completa de shelters (de 80 a 1000 assinantes) com 13.500 armários remotos de comutação instalados em campo (Brasil, China, Alemanha, Polônia) resultando em, aproximadamente, 6,5 milhões de assinantes. Desde 1997, o Centro Regional de Desenvolvimento no Brasil é responsável pelos projetos dos clientes na América Latina e, atualmente, é um dos onze Regional Development Centers (RDCs) mundiais para desenvolvimento de software para New Generation Networks (NGN), bem como um dos dois centros mundiais de hardware para equipamento de controle NGN e também centro de competência para a América Latina no desenvolvimento de software para essa tecnologia.

$\mathrm{Na}$ área de comunicação privada, tem 9 centros de desenvolvimento no mundo e o Brasil é um deles. Essa área consolidou-se como um centro de competência mundial para equipamentos de PABX de pequeno e médio porte. Também passou a ser base de exportação mundial de produtos, inclusive fornecendo para os EUA.

A área de comunicação móvel está se consolidando como centro de competência também para o segmento de telefonia celular; as estações rádio base foram modificadas para atender às exigências do mercado brasileiro; e na área de telefones celulares desenvolve-se software para terminais móveis em parceria com universidades e centros de pesquisa.

Nesse contexto, os centros de pesquisa e desenvolvimento e as unidades fabris brasileiros ocupam lugar de destaque na organização. A fábrica de Manaus, por exemplo, constitui um dos três centros de competência mundiais da Siemens para a fabricação dos telefones celulares GSM, e a fábrica em Curitiba, hoje é base de exportação de centrais de comutação públicas, móveis e privadas, para estas últimas, constituindo o chamado pólo de exportação mundial.

Tabela 1 - Unidades Industriais.

\begin{tabular}{|c|c|c|}
\hline ESTADO & CIDADE & TIPO DE PRODUTO \\
\hline Amazonas & Manaus & Aparelho telefônico celular e convencional \\
\hline Paraná & Curitiba & Centrais telefônicas públicas e privadas e Estações Rádio Base \\
\hline
\end{tabular}

Fonte: Siemens.

Revista Ibero-Americana de Estratégia - RIAE, São Paulo, v. 10, n. 3, p. 173-198, set./dez. 2011. 
Milton Freitas Chagas Junior, Roque Rabechini Jr, Arnoldo Souza Cabral \& Milton de Abreu Campanario

Tabela 2 - Centros de Pesquisa \& Desenvolvimento.

\begin{tabular}{|c|c|}
\hline LOCAL & TIPO DE DESENVOLVIMENTO \\
\hline Curitiba/PR & Comunicação e transmissão para operadoras fixas e móveis. \\
\hline Curitiba/PR & Comunicação privada para o segmento corporativo. \\
\hline Curitiba/PR & Telefones analógicos. \\
\hline Manaus/AM & Telefones móveis celulares. \\
\hline
\end{tabular}

Fonte: Siemens.

\section{APRESENTAÇÃo E ANÁLISE DOS RESULTADOS}

A estratégia tecnológica de organizações que lideram processos de inovação de produtos multitecnológicos é centrada na manutenção de grupos especializados que acompanham a evolução das disciplinas que formam a base de conhecimentos tecnológicos de seus produtos, permitindo e fomentando a criação e descoberta de soluções inovadoras através da integração dessas disciplinas. Essas organizações coordenam o processo de aprendizado, de forma dispersa. Existe, portanto, diferentes graus de especialização entre unidades organizacionais baseadas nas disciplinas tecnológicas que devem dominar para coordenar os processos de inovação.

Foram escolhidas as empresas Embraer e Siemens Brasil como exemplo de indústrias multitecnológicas que lideram redes globais de produção, pelas características de suas capacitações: necessidade de domínio de uma base ampla de conhecimentos tecnológicos, formada por diversas disciplinas que avançam a taxas diferenciadas e mostram padrões de interdependências de elevada complexidade. A natureza dessas capacitações permite-nos analisar a natureza dos relacionamentos, e o consequente nível de acoplamento interorganizacional definido pelas firmas líderes com sua rede de fornecedores especializados, fundamentalmente em aspectos relacionados com a integração de conhecimento tecnológico.

Na Embraer foi analisada a família Embraer 170/190. No desenvolvimento dessa família, nota-se uma redução significativa das atividades de projeto detalhado e da produção de módulos realizados diretamente pela Embraer.

$\mathrm{Na}$ Siemens Brasil foram analisados dois produtos de duas unidades de negócio distintas: equipamento de raios- $\mathrm{X}$ da unidade de eletromedicina e a plataforma de redes de dados móveis SX 
da unidade de comunicação móvel. Nesses produtos, assim como no caso da família Embraer 170/190, nota-se uma redução das atividades de projeto detalhado e produção, comparativamente ao que se faziam nos produtos de geração anteriores da Siemens.

\section{a. Embraer 170/190: rede de acoplamento frouxo}

A Embraer estabeleceu e lidera a rede de desenvolvimento do programa Embraer 170/190 dos pontos de vista tecnológico e organizacional. A rede de fornecedores foi estabelecida em torno da arquitetura modular das aeronaves dessa família. Muitos dos fornecedores especializados de primeiro nível atuam como parceiros de risco do programa.

Embora haja uma forte tendência de desintegração da produção e do projeto detalhado, existe grande superposição de conhecimentos tecnológicos entre a firma integradora de sistemas e outros agentes da rede. Assim, a forma de rede mais adequada para o caso da indústria aeronáutica é a coordenação do projeto realizada por meio de acoplamento frouxo, pois combina o conceito de interdependência sistêmica imprevisível com aquele de taxas de mudanças tecnológicas de componentes constantes. Cabe ressaltar que as taxas estáveis de mudança se referem ao hardware dos módulos, pois o software, que faz a interface entre módulos, avança a taxas inconstantes e os esforços de $\mathrm{P} \& \mathrm{D}$, desenvolvimento e produção tendem a ser mantidos internamente, caracterizando uma situação de acoplamento firme. A figura 2 abaixo representa a estratégia de modularização da rede utilizando o acoplamento frouxo como mecanismos de coordenação.

Figura 2- Divisão do trabalho em módulos entre parceiros de risco - Embraer 170.

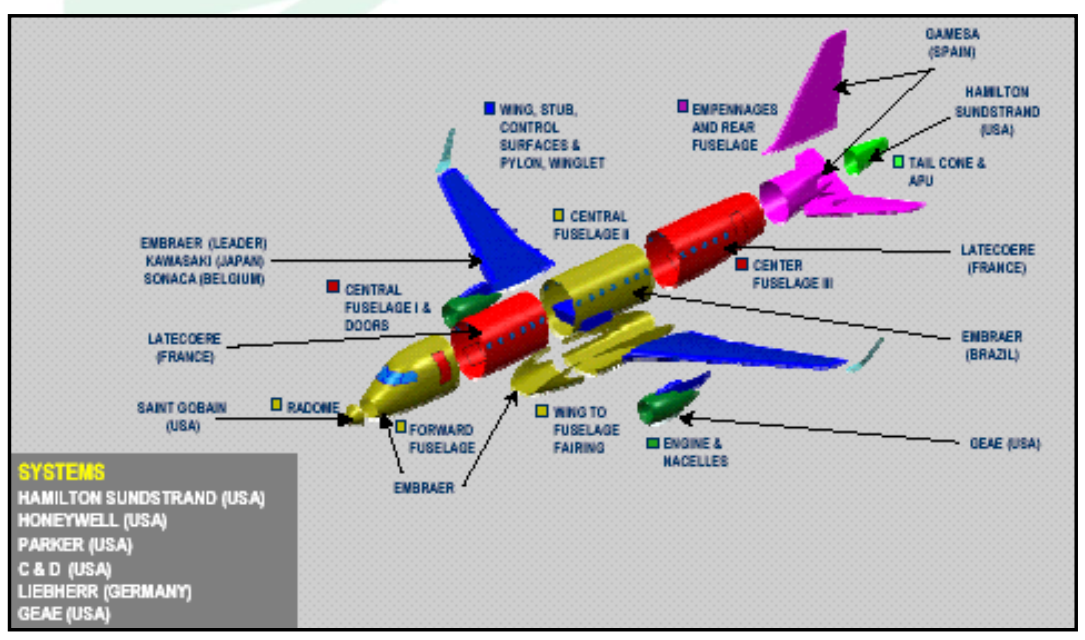

Fonte: Embraer.

Revista Ibero-Americana de Estratégia - RIAE, São Paulo, v. 10, n. 3, p. 173-198, set./dez. 2011. 
No caso do desenvolvimento do Embraer 170/190, as interdependências sistêmicas do produto - caracterizadas pelas imprevisibilidades e não-linearidades - exigem que a rede tenha disponibilidade de recursos de $\mathrm{P} \& \mathrm{D}$ em excesso, permitindo a absorção de perturbações de origem interna e externa ao longo do desenvolvimento do produto. As capacitações para conceber, controlar o desenvolvimento de módulos e se responsabilizar pela integração final das aeronaves estão fundamentadas no domínio, pela firma líder, de uma base tecnológica de amplitude crescente.

Os critérios para a seleção de parceiros são múltiplos e dependentes, inclusive, de fatores relacionados a política de comércio exterior dos países. Por exemplo: um fator que influencia na definição dos parceiros externos é a possibilidade de aumentar a penetração em mercados externos, pois as companhias aéreas dão preferência para produtos em que existam parceiros de mesma nacionalidade. Um outro critério utilizado é selecionar as firmas que estejam dispostas a investir antecipadamente, trazendo liquidez, ao programa. Para programas de desenvolvimento com períodos longos de maturação, ter liquidez nas fases iniciais se constitui num fator crítico, que, muitas vezes, pode determinar sua viabilidade financeira. Os fatores principais, no entanto, são suas capacitações e a disposição de atender às especificações e as inevitáveis alterações que, ao longo do ciclo de vida do produto, serão definidas pela firma líder.

Os contratos estabelecidos com os parceiros seguem as cláusulas gerais de um contratomestre do produto, o Master Product Contract (MPC), e outras específicas para cada contrato. Uma cláusula padrão em todos os contratos com fornecedores é a possibilidade da Embraer solicitar modificações nos módulos de seus parceiros para atender aos requisitos de certificação da aeronave - certificado de homologação de tipo CHT. Esses contratos abrangem todo o ciclo de vida do produto e o relacionamento entre os agentes não é baseado exclusivamente em critérios de preços, mas consideram também aspectos como confiança e reciprocidade, o que permite a redução dos custos de transação. Essa é uma forma de reduzir a incerteza técnica e comercial do produto e indica que o mecanismo de coordenação mais apropriado para representar esses relacionamentos entre os agentes seja a integração de sistemas, na medida em que mescla características do sistema de preços e da integração vertical.

\section{b. Siemens}

Interpretaremos, utilizando a estrutura conceitual apresentada na seção 2, as diferenças entre as fronteiras do conhecimento e da produção de duas unidades de negócio da Siemens Brasil representadas por produtos desenvolvidos no Brasil. O desenvolvimento desses produtos exigiu o estabelecimento de relacionamentos de longo prazo, que vêm propiciando grande interação entre os

Revista Ibero-Americana de Estratégia - RIAE, São Paulo, v. 10, n. 3, p. 173-198, set./dez. 2011. 
agentes, incluindo unidades de negócio de outros países, fornecedores e parceiros. Os produtos são: equipamento de raios-X da unidade de eletromedicina e plataforma de redes de dados móveis SX da unidade de comunicação móvel.

\section{i. Equipamento de raios - X: Rede de acoplamento frouxo}

A unidade de negócios de eletromedicina representa apenas 3\% do faturamento da Siemens, mas é a área que vem mais trabalhando na busca de novas oportunidades de negócio na região Mercosul, com o intuito de ampliar sua participação de mercado. Especificamente, o equipamento low-end de raios- $\mathrm{X}$ foi desenvolvido para atender a demanda do mercado latino-americano, com disponibilidade de pagar significativamente menor que os mercados americano e europeu.

Assim como no caso aeronáutico, a regulamentação é um fator decisivo nessa indústria, pois somente é permitida a entrada de produtos médicos no mercado, após serem aprovados pela Agência Nacional de Vigilância Sanitária (Anvisa). A competência para especificar o produto que atenda de forma abrangente à demanda latino-americana juntamente com a capacidade de atender aos requisitos de certificação do produto são fatores críticos para a inserção do produto no mercado.

Conforme mostrado na figura 3, o equipamento é composto por cinco módulos: mesa e stand vertical, gerador de alta frequência, grid, colimador e tubos de raios-X. Os principais fornecedores locais produzem a mesa e o gerador de alta frequência, sendo os demais módulos desenvolvidos e manufaturados por unidades organizacionais da Siemens na Alemanha e EUA. Essa é a forma de relacionamento interorganizacional de acoplamento frouxo, pois combina o conceito de interdependência sistêmica imprevisível com taxas de mudanças tecnológicas constantes.

Uma característica específica do integrador de sistemas é a coordenação dos trabalhos de fornecedores da rede, contratando projeto detalhado e produção de fornecedores especializados, mantendo o domínio de projeto conceitual e a capacidade de integração. O relacionamento entre a firma integradora de sistemas e seus fornecedores é regido por contratos que abrangem desde uma simples prestação de serviços de manutenção pós-venda até o as linhas mestras das parcerias para minimização de riscos técnicos e comerciais.

A estratégia de desenvolvimento do produto é baseada na arquitetura modular, que exige a coordenação de mudanças de um grande espectro de tecnologias, que, por sua vez, requerem o domínio de uma miríade de conhecimentos. A terceirização de projeto detalhado e a produção de alguns módulos não implicam a perda do domínio de conhecimentos tecnológicos específicos.

Revista Ibero-Americana de Estratégia - RIAE, São Paulo, v. 10, n. 3, p. 173-198, set./dez. 2011. 
É importante ressaltar que as capacitações para especificar, realizar a integração final e testes exigidos para a certificação englobam a base de conhecimentos que fundamentam o produto.

Nesse caso, as tecnologias mais sensíveis estão nos módulos fornecidos por outras unidades de negócio da própria Siemens e pode-se considerar uma situação de acoplamento firme, na medida em que o mecanismo de coordenação é mais bem representado pela integração vertical, embora os interesses das unidades de negócio nem sempre sejam coincidentes.

Figura 3 - Divisão do trabalho em módulos entre parceiros - Raio X Siemens.

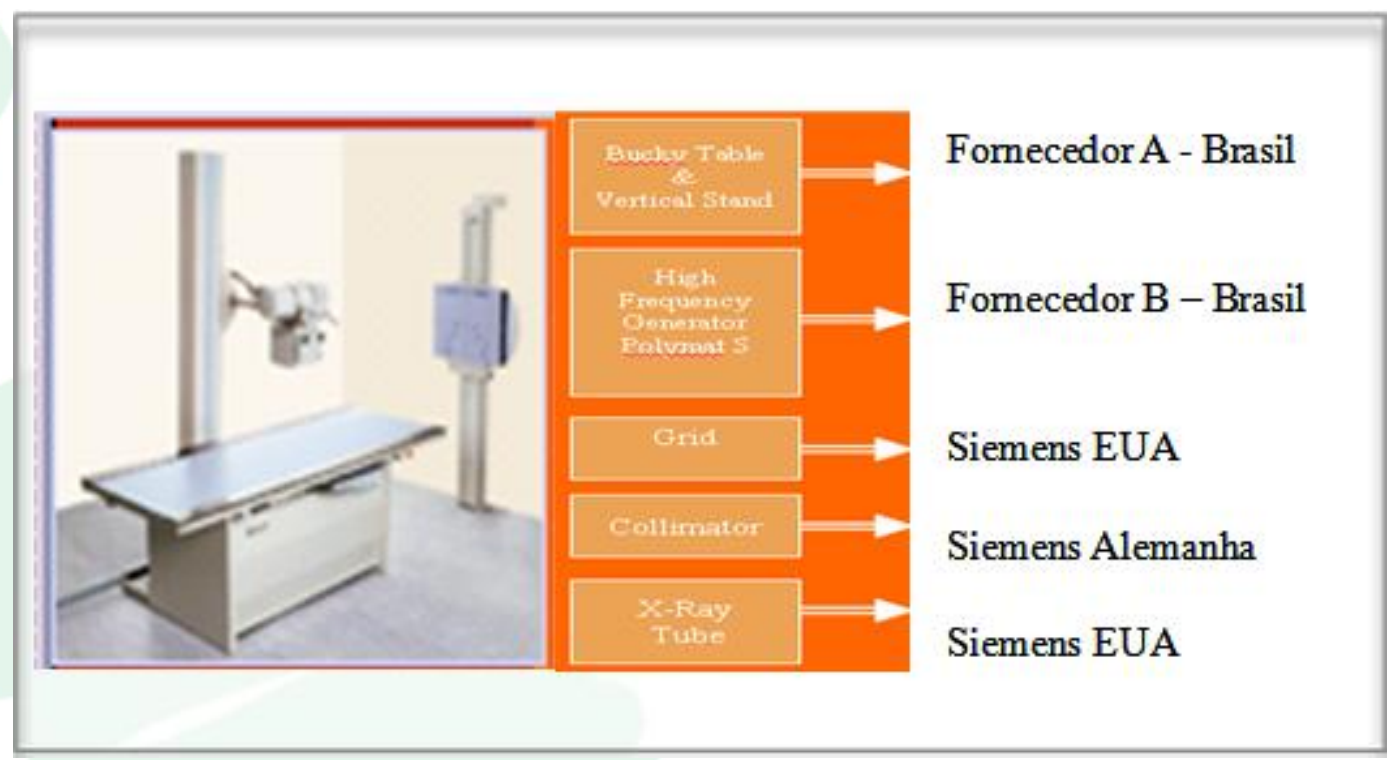

Fonte: Siemens.

\section{ii. Plataforma SX: Transição de acoplamento forte para frouxo}

A unidade de negócios de comunicações móveis da Siemens Brasil está se consolidando como centro de competência para o segmento de telefonia celular desde que foi introduzida a tecnologia GSM no mercado brasileiro.

Especificamente, o SX é uma plataforma, composta por diversos módulos, que gerencia e contabiliza o tráfego de serviços de mensagens e comunicação wireless, entre sistemas corporativos e dispositivos móveis (celulares, pagers, PDAs, notebooks, etc), a princípio atendendo o tráfego Short Message Service (SMS) e evoluindo para o tratamento de pacote de dados multimídia (MMS).

Para atender com mais eficiência e abrangência o mercado, a estratégia de desenvolvimento foi baseada na modularidade. $\mathrm{O}$ processo de desenvolvimento da plataforma envolveu equipes multidisciplinares de especialistas nas áreas de arquitetura de software, designers, desenvolvimento de software e integração e testes.

Revista Ibero-Americana de Estratégia - RIAE, São Paulo, v. 10, n. 3, p. 173-198, set./dez. 2011. 
Esse é o exemplo de relacionamento interorganizacional que deveria ser enquadrado como acoplamento firme, pois combina o conceito de interdependência sistêmica imprevisível com aquele de taxas de mudança tecnológica de componentes inconstantes.

As elevadas taxas de mudança técnica do setor de comunicações, especificamente software (protocolos) e microeletrônica, tendem, inicialmente, a manter o máximo da capacitação tecnológica internamente. A Siemens Brasil possui hoje as capacitações de concepção de arquitetura, desenvolvimento, produção e comercialização do espectro completo de produtos e sistemas de telefonia móveis, bem como atividades de prospecção de oportunidades tecnológicas geradas pelas universidades e institutos de pesquisa brasileiros (Cavalcante, 2004).

No entanto, o efeito da acumulação de capacitações em projetos dessa natureza permitiu que a Siemens Brasil estabelecesse parcerias com instituições científicas e tecnológicas brasileiras localizadas em São Paulo, Curitiba e Recife - para a execução de módulos de sua plataforma, uma vez que o grau de imprevisibilidade das interdependências do produto foi progressivamente sendo reduzido, em razão da experiência adquirida na execução de outros projetos dessa natureza.

O principal diferencial da Plataforma SX é sua conectividade, permitindo que, independentemente das operadoras de telecomunicações móveis, os usuários finais possam receber ou enviar SMS, MMS ou mesmo e-mail. A arquitetura da plataforma é modular, conforme descrito na figura 4.

Revista Ibero-Americana de Estratégia - RIAE, São Paulo, v. 10, n. 3, p. 173-198, set./dez. 2011. 
Milton Freitas Chagas Junior, Roque Rabechini Jr, Arnoldo Souza Cabral \& Milton de Abreu Campanario

Figura 4 - Arquitetura modular da Plataforma SX - Siemens.

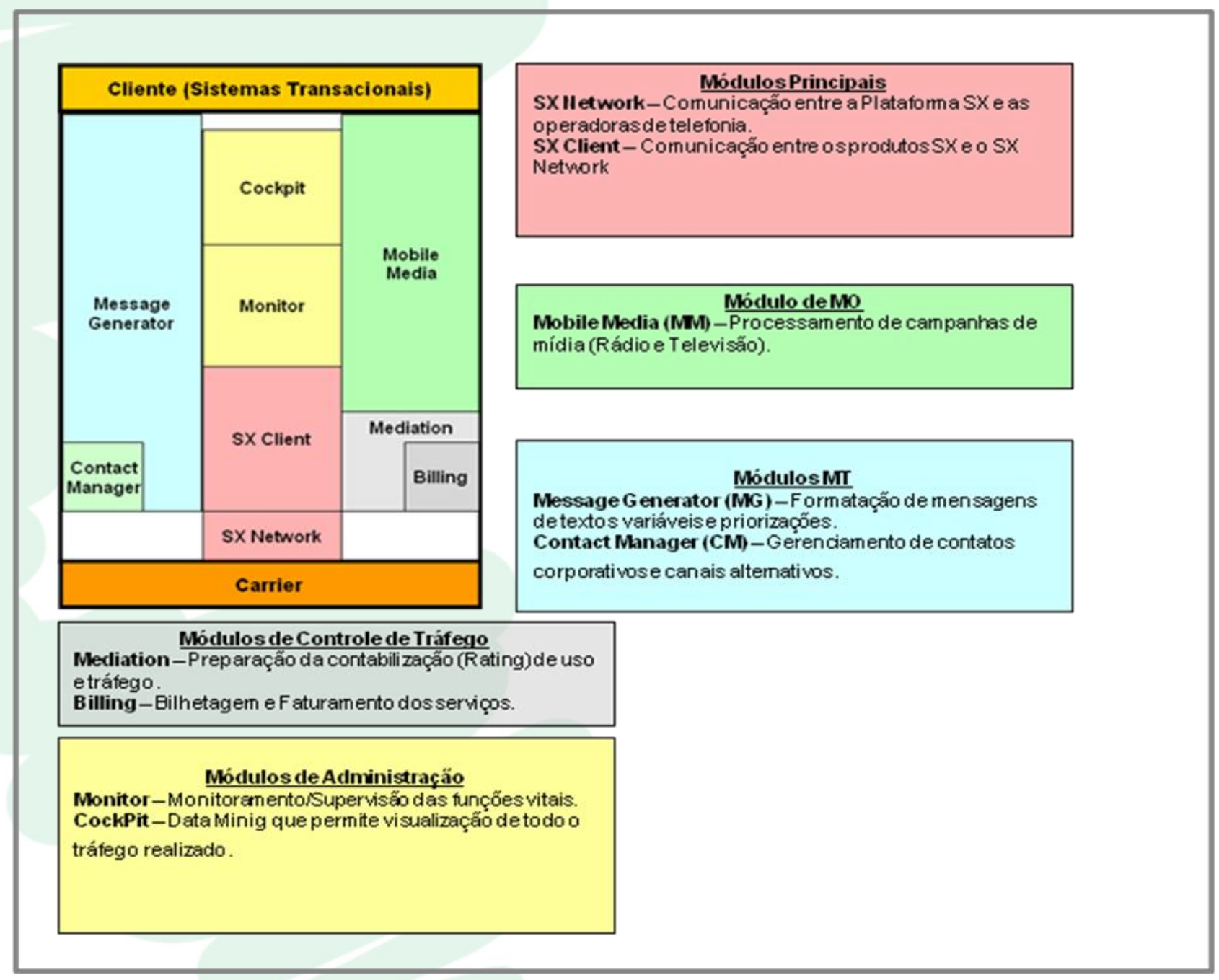

Fonte: Siemens.

Ressalta-se que com o objetivo de tornar o produto mais competitivo, a estratégia de desenvolvimento seguiu a estratégia da modularidade, por meio do estabelecimento de parcerias com fornecedores especializados externos, que vieram a complementar e auxiliar os esforços internos de desenvolvimento e produção dos módulos. Com as contratações estabelecidas dessa forma, foi possível a redução de custos, riscos e períodos de desenvolvimento. Assim, atividades que, há bem pouco tempo, eram realizadas internamente, com recursos exclusivos da Siemens, passaram a ser contratadas externamente.

Revista Ibero-Americana de Estratégia - RIAE, São Paulo, v. 10, n. 3, p. 173-198, set./dez. 2011. 


\section{c. Fechamento dos casos}

Os relacionamentos interorganizacionais observados nas apresentações dos casos foram enquadrados segundo o modelo de Brusoni, Prencipe e Pavitt (2001) nas categorias de acoplamento frouxo e firme, conforme representada na figura 7.

Figura 5- Transição da forma de acoplamento interorganizacional, em razão da capacidade de predição do comportamento do produto de plataformas móveis.

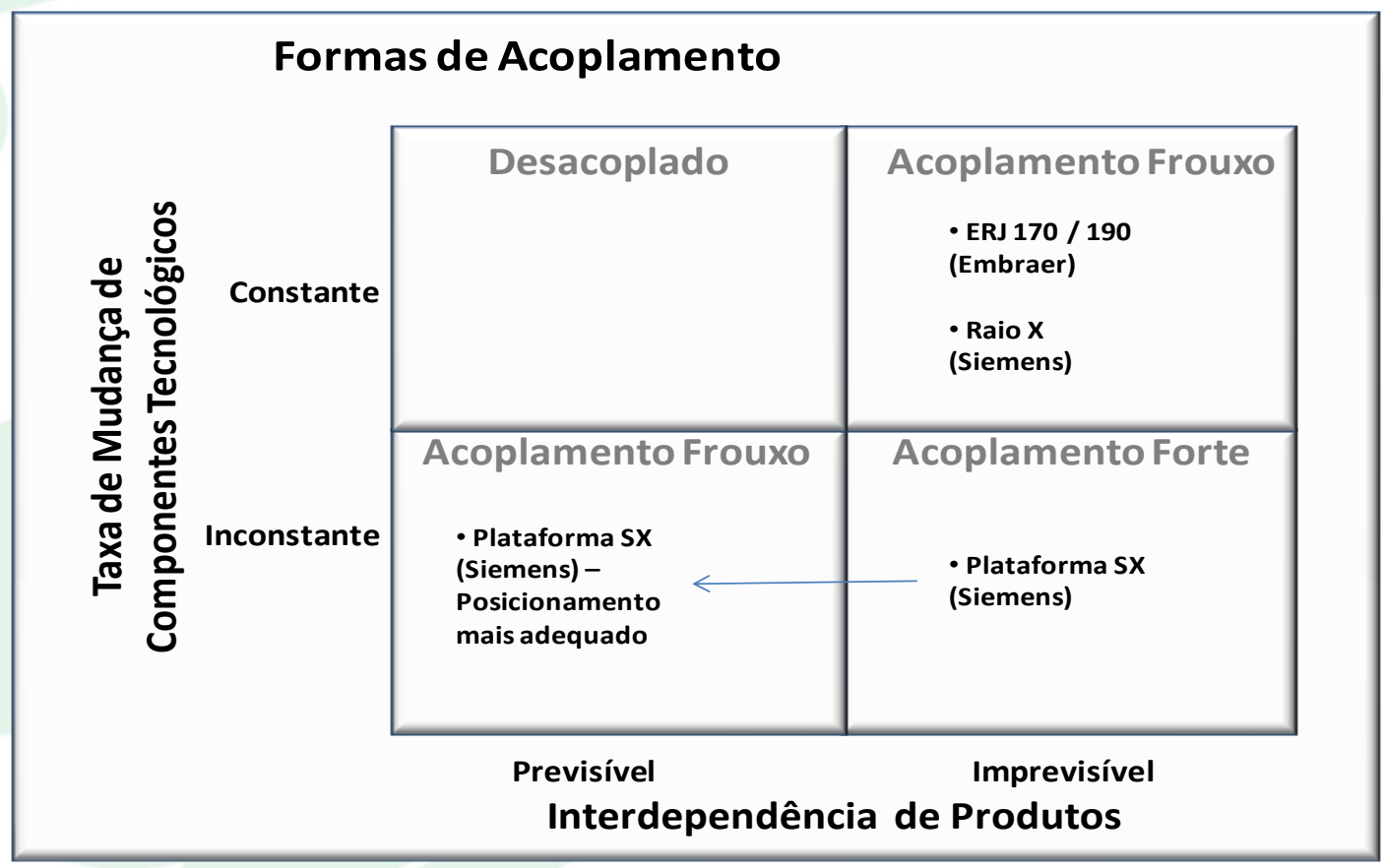

Fonte: Adaptado de Brusoni, Prencipe e Pavitt (2001).

O acoplamento frouxo foi observado nos casos da Embraer (jatos da família ERJ170 / 190) e da Siemens (Raio X). Nesses casos, foi possível perceber que a interdependência dos produtos se dá de forma imprevisível e que pela dinâmica das tecnologias envolvidas existe uma taxa constante de mudança em seu desenvolvimento.

Foi possível verificar também a existência de um produto (Siemens Plataforma SX) com características de acoplamento forte observado pelas informações de imprevisibilidade, em termos de interdependência dos produtos, e com taxas de mudanças tecnológicas com comportamento inconstante.

Nesse caso, em particular, se vê que a empresa pode se organizar em forma de acoplamento frouxo, organizando a interdependência de produto. Essa informação pode ajudar a empresa melhor se estruturar para responder ao mercado e aos seus custos.

Revista Ibero-Americana de Estratégia - RIAE, São Paulo, v. 10, n. 3, p. 173-198, set./dez. 2011. 


\section{CONSIDERAÇÕES FINAIS}

As redes interorganizacionais, como alternativas estruturais, foram analisadas nesse trabalho por meio de dois casos representativos desse tipo organizacional, utilizando modelo conceitual proposto por Brusoni, Prencipe e Pavitt (2001). Assim sendo, foi possível expandir a compreensão sobre os mecanismos de coordenação de redes interorganizacionais estabelecidas para o desenvolvimento e produção em indústrias multitecnológicas. Por meio de evidência empírica, foram feitos estudos de caso de produtos da Embraer e da Siemens Brasil, que atuam como firmas integradoras de sistemas, estabelecendo e liderando redes de fornecedores especializados, dos pontos de vista tecnológico e organizacional, em torno de plataformas de produto de arquitetura modular. Em particular, foi ressaltado que os mecanismos de coordenação que melhor caracterizam o relacionamento entre a firma líder e sua rede de fornecedores é a integração de sistemas, que mescla características das formas tradicionais de coordenação, o sistema de preços e a integração vertical. Para se manterem competitivas, as firmas desenvolvem e mantém capacitações em integração de sistemas. Tais capacitações dão a elas a possibilidade de integrar componentes e conhecimentos tecnológicos desenvolvidos, tanto interna quanto externamente às suas fronteiras.

O trabalho mostrou ainda que as organizações que lideram as redes de inovação não são simplesmente montadoras de componentes físicos, mas são melhores caracterizadas como integradoras de conhecimento. Essas organizações desempenham papel fundamental na definição do conceito do produto, na sua divisão em módulos e na integração de sistemas. Eles mantêm internamente a capacitações para a elaboração conceitual a partir da identificação de novas oportunidades de negócios, para dividir o projeto em módulos, para coordenar a rede de fornecedores especializados de primeiro nível e então recompõe o produto numa dada arquitetura, por meio da integração de seus módulos. Dessa forma, suas atividades de P\&D não são focadas. Ao contrário, suas capacitações devem ser amplas, abrangendo toda base de conhecimentos tecnológicos que fundamentam seus produtos. $\mathrm{O}$ trabalho traz evidências que existe c imbricação entre as capacitações das firmas lideres e a aquelas dos fornecedores especializados. Portanto, a modularidade do produto não implica a modularidade do conhecimento tecnológico, pois existem limites cognitivos para a divisão do conhecimento. A definição das fronteiras da firma proposta por Willianson, em termos das decisões entre fazer ou comprar, estão muito mais relacionadas com as atividades produtivas do que com as capacitações tecnológicas mantidas internamente. Na realidade, não existe superposição entre as fronteiras relacionadas com atividades produtivas e as com domínios da base de conhecimentos que fundamentam o produto. São essas capacitações em

Revista Ibero-Americana de Estratégia - RIAE, São Paulo, v. 10, n. 3, p. 173-198, set./dez. 2011. 
excesso, que geram capacidade de inovar e absorver tecnologias desenvolvidas exogenamente às organizações, que permitem que os custos de transação possam ser reduzidos de forma consciente pelas firmas integradoras de sistemas, tornando seus produtos mais competitivos.

\section{REFERÊNCIAS}

Afuah(2001) A. Dynamics boundaries of the firm: are firms better off being vertically integrated in the face of technological change? Academy Of Management Journal, 44(6),1211-1228.

Arora, A., Gambardella, A., Rullani, E.(1998) Division of labour and the locus of inventive activity, Journal of Management and Governance, 1, 1-19.

Brusoni, S., Pavitt, K. , Prencipe, A.(2001) Knowledge specialization, organizational coupling and the boundaries of the firm: why do firms know more than they make? Administrative Science Quarterly, 46, 597-621.

http://dx.doi.org/10.2307/3094825

Carvalho, M. M., Rabechini Jr, R. (2007) Construindo competências em gerenciamento de projetos: teoria e casos.(2.Ed). Editora Atlas.

Chagas Junior M.F, Cabral, A.S, Campanário, M A.(2011) Firmas integradoras de sistemas, suas capacitações e fontes de tecnologia. O caso da Embraer. In: Revista de Ciências da Administração V. 13, N.29, 63 - 87.

Chagas Junior, M.F.(2005) A evolução dos modelos de gestão do processo de inovação tecnológica nas firmas: o caso da Embraer, Tese de Mestrado - Instituto Tecnológico de Aeronáutica - ITA, São José dos Campos, SP.

Cleland, D. I., Ireland, L. R.(2007) Gerenciamento de projetos, revisão técnica. (2.a edição) Rio de Janeiro, LTC.

Coase, R. (1937) The nature of the firm, Economica, 4, 386-405.

http://dx.doi.org/10.1111/j.1468-0335.1937.tb00002.x

Cavalcante, M.B. (2004) Portal de tecnologias da siemens do brasil: instrumento para alavancar a máquina de inovação de empresa multinacional intensiva em conhecimento, In: Anais do Simpósio de Gestão de Inovação Tecnológica - PGT/USP., 2165 - 2178 Curitiba, Paraná, Brasil, 23.

Revista Ibero-Americana de Estratégia - RIAE, São Paulo, v. 10, n. 3, p. 173-198, set./dez. 2011. 
Deresky, H.(2004) Administração global - estratégica e interpessoal. Porto Alegre, Bookman,. Capítulo 8 - Organização da Estrutura, dos sistemas de controle e das relações trabalhistas, 203233.

Dosi, G. (1988) Sources, procedures, and microeconomic effects of innovation. Journal of Economic Literature, 26(3), 1120 - 1171.

Eisenhardt, K. M. (1989) Building theories from case study research. Academy of Management Review, Briar Cliff Manor, 14(4), 532-550.

Fleury, M.T. , Fleury, A. (eds.). (2004) Política Industrial, São Paulo, Publifolha, 1,2.

Gray, C.F., Larson, E. W.(2009) Gerenciamento de projetos: o processo gerencial, ISBN: 978-857726-064-5, São Paulo, McBooks.

Henderson, R. M., Clark, K. B.(1990) Architectural innovation: the reconfiguration of existing product technologies and the failure of established firms, Administrative Science Quarterly, 35, 9-30.

http://dx.doi.org/10.2307/2393549

Kerzner, H.(2001) Applied project management best practices on implementation. New York: John Wiley \& Sons.

Kogut, B. (Mar 2000) The network as knowledge: generative rules and the emergence of structure. Strategic Management Journal, 21, 3; ABI/INFORM Global.

http://dx.doi.org/10.1002/(SICI)1097-0266(200003)21:3<405::AID-SMJ103>3.0.CO;2-5

Lawrence, P.R., Lorsch, J. W.(1967) Organization and environment: managing differentiation and integration. Homewood: R. D. Irwin.

Mintzberg, H., Ahlstrand, B. E Lampel, J. (2000) Safári de estratégia: um roteiro pela selva do planejamento estratégico. Porto Alegre: Bookman.

Patah, L. Carvalho, M.M. (2002) Estruturas de gerenciamento de projetos e competências em equipes de Projetos. In: Encontro Nacional de Engenharia de Produção, ENEGEP Curitiba, Brasil, 22.

Perrow, C. A. (Apr. 1967) framework for comparative organizational analysis. American Sociological Review, Washington, 32(2),194-208.

Rosenberg, N.(1976) Perspectives on technology, Cambridge, Cambridge University Press. http://dx.doi.org/10.1017/CBO9780511561313

Revista Ibero-Americana de Estratégia - RIAE, São Paulo, v. 10, n. 3, p. 173-198, set./dez. 2011. 
Rothwell, R. (1992) Successful industrial innovation: critical factors for the 1990s, $R \& D$ Management, 22/3, 221-39.

http://dx.doi.org/10.1111/j.1467-9310.1992.tb00812.x

Rodan, S., Galunic, C. (Jun. 2004) More than network structure: how knowledge heterogeneity influences managerial performance and innovativeness. Strategic Management Journal. ABI/INFORM Global, 25(6), 541,.

Shenhar, A. J. (Mar. 2001) One size does not fit all projects: exploring classical contingency domains. Management Science, Providence, 47(3), 394-414.

Teece D. J.(1996) Firm organization, industrial structure, and technological innovation, Journal of Economic Behavior \& Organization, 31(2), 193-224.

http://dx.doi.org/10.1016/S0167-2681(96)00895-5

Thompson, J. D. (1967) Organizations in action. New York: McGraw-Hill.

Vasconcellos, E., Hemsley, Jr. (2002) Estruturas organizacionais - estruturas tradicionais, estruturas para inovação, estrutura matricial. São Paulo, Editora Thomson Pioneira (4a edição), $3-16$.

Williamson, O. E. (1996). The mechanisms of governence, Oxford.

Yin, R. (2001) Estudo de caso: planejamento e métodos. Trad. de Daniel Grassi.(2.ed.) Porto Alegre: Bookman.

Recebido: 13/07/2011

Aprovado: 04/10/2011

Revista Ibero-Americana de Estratégia - RIAE, São Paulo, v. 10, n. 3, p. 173-198, set./dez. 2011. 